\section{Comprendre la CRDS pulsée}

Nathalie LAMOUREUX

Laboratoire Physico-chimie des Processus de la Combustion et de l'Atmosphère, PC2A, UMR 8522 Lille 1/CNRS

nathalie.lamoureux@univ-lille 1.fr

L'acronyme CRDS signifie cavity ring down spectroscopy et peut être traduit en français par «spectroscopie à temps de déclin d'une cavité optique » (ou spectroscopie par temps d'amortissement de résonance, STAR [1]). Cette technique, initialement dédiée à la mesure des coefficients de réflexion de miroirs, a été appliquée pour la première fois par $\mathrm{O}^{\prime} \mathrm{Keefe}$ et Deacon en 1988 à la mesure par spectroscopie d'absorption d'une espèce moléculaire avec une sensibilité de $10^{-6}$ par $\mathrm{cm}$ [2]. Depuis, des variantes de la technique ont été proposées ; cw-CRDS (continuous wave CRDS), BB-CEAS (broad band cavity enhanced absorption spectroscopy), PS-CRDS (phase shiftCRDS), ICLAS (intra cavity laser absorption)... Dans cet article, nous présentons le principe de la CRDS pulsée et quelques applications aux mesures en combustion.

\section{Absorption/absorbance}

La technique CRDS est une méthode d'absorption et repose sur la loi de BeerLambert qui établit une relation de proportionnalité entre l'absorption d'un faisceau de lumière monochromatique dans un milieu homogène et isotrope, le trajet optique du faisceau et la concentration d'une espèce selon l'expression :

$$
I_{t}=I_{0} \exp (-\alpha \ell)
$$

$I_{0}$ et $I_{\mathrm{t}}$ sont respectivement les intensités incidente et transmise du faisceau, $\ell$ est la longueur du trajet optique. $\alpha$ (exprimé en $\mathrm{cm}^{-1}$ ) est le coefficient d'absorption du milieu qui est égal au produit de la section efficace d'absorption $\sigma$ (en $\mathrm{cm}^{-1}$ / (molécule $\mathrm{cm}^{-3}$ )) et de la concentration de l'espèce absorbante (en molécule $\mathrm{cm}^{-3}$ ). Les termes $\sigma$ et $\alpha$ dépendent de la longueur d'onde du faisceau et de la température du milieu. Cependant, la loi de Beer-Lambert n'est vérifiée que si le milieu est optiquement mince, c'est-à-dire lorsque $\alpha \ell<<1$. La méthode d'absorption la plus simple consiste à mesurer l'atténuation du signal lumineux lors de son trajet dans le milieu absorbant de longueur $\ell$, comme le montre la figure 1. Cela suppose de pouvoir mesurer avec précision une différence d'intensité de lumière, sachant qu'il est souvent délicat d'avoir une source de lumière parfaitement stable en intensité. L'augmentation de la sensibilité de la technique passe par l'augmentation du trajet optique, soit en augmentant la longueur du dispositif, soit en utilisant des miroirs qui vont permettre d'accroître le trajet optique en multipliant le nombre de réflexions de la lumière dans la cellule de mesure (absorption multi-passage). Dans ces conditions, l'atténuation d'intensité mesurable est de l'ordre de $10^{-3}$, soit une sensibilité de $10^{-3}$ par $\mathrm{cm}$.

\section{NOUVEAU}

Luxmètre CL-70F

Sources lumineuses et LED

Précision et Résolution

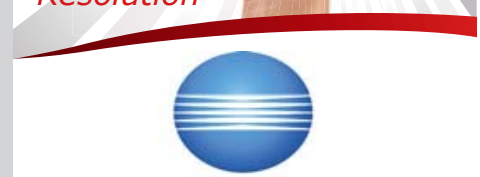

KONICA MINOLTA

\section{Mesure \& évaluation}

- Éclairement lumineux

Température de couleur

Indice de Rendu des Couleurs (IRC)

Spectre

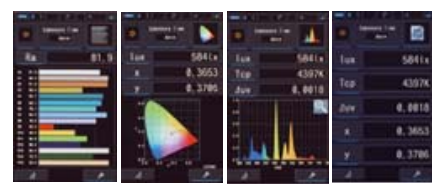

Facile d'utilisation

Grand écran couleur

Portable \& tactile

Personnalisable

Logiciel inclus

Récepteur rotat $\left(180^{\circ}\right)$

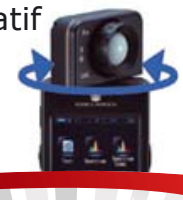

ScienTec c'est aussi...

Spectroradiomètres

Vidéo-colorimètres

Photomètres

Luxmètres

Chromamètres

Sources de référence

info@scientec.fr / www.scientec.fr 01.64.53.27.00 


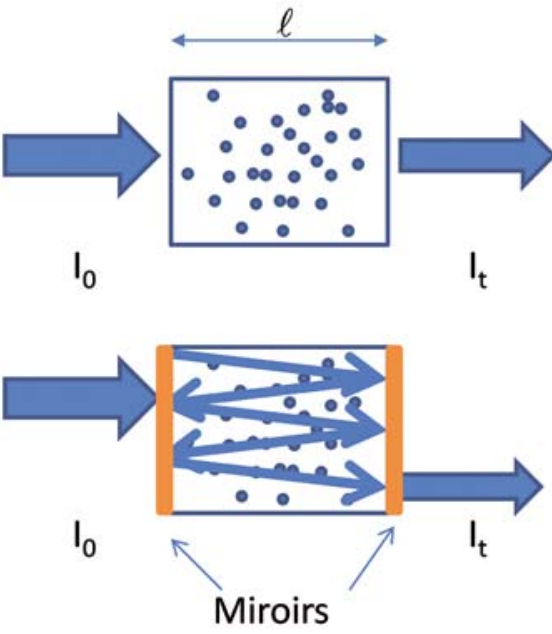

\section{Absorption multi-passage}

\section{Absorption simple passage}

\section{Figure 1. Principe de méthodes d'absorption.}

\section{Principe général de la CRDS}

Le principe de la CRDS repose sur la mesure de la vitesse de déclin de l'intensité de la lumière dans une cavité optique, plutôt que sur l'amplitude d'absorption. De façon simple, la CRDS consiste à mesurer le temps de vie d'une impulsion laser qui effectue de très nombreux allers et retours entre les deux miroirs hautement réfléchissants de la cavité optique (coefficient de réflexion, $R$ ). L'intensité du pulse décroît dans la cavité du fait de l'absorption des molécules et des pertes à chaque réflexion sur les miroirs. À chaque fois que l'impulsion est réfléchie, une faible fraction de l'intensité est transmise $\left(I_{1}, I_{2}, \ldots, I_{n}\right)$. Après $n$ allers-retours, l'intensité transmise s'exprime selon :

$$
I_{n}=I_{0} R^{2 n} \exp (-2 n \alpha \ell)
$$

Un détecteur placé en sortie de la cavité permet de mesurer l'intensité transmise en fonction du temps comme le montre la figure 2. On peut alors remplacer la valeur discrète $n$ par le temps rapporté à $t=n 2 d / c$ (c étant la célérité de la lumière, $\mathrm{d}$ la distance entre les miroirs). Compte tenu du fait que le coefficient de réflexion des miroirs est proche de 1 $(\ln R \approx-(1-R))$, la décroissance temporelle de l'intensité peut s'exprimer selon une loi exponentielle :

$$
I(t)=I_{0} \exp \left(-\frac{t c}{d}(1-R+\alpha \ell)\right)=I_{0} \exp \left(-\frac{\mathrm{t}}{\tau}\right)
$$

La CRDS repose donc sur la mesure du temps de déclin ou temps de vie de l'impulsion laser dans la cavité, $\tau$. En absence d'absorption dans la cavité $(\alpha=0)$, le temps de vie s'exprime selon :

$$
\tau_{0}=\frac{d}{c[(1-R)]}
$$

La présence d'un milieu absorbant dans la cavité $(\alpha \neq 0)$ entraîne l'augmentation de la vitesse de déclin de l'impulsion et $\tau$ s'exprime selon :

$$
\tau=\frac{d}{c[(1-R)+k]}
$$

L'absorbance ( $k=\alpha \ell$ ) peut être déterminée par comparaison des inverses des temps de vie mesurés respectivement avec et sans absorption :

$$
k=\frac{d}{c}\left(\frac{1}{\tau}-\frac{1}{\tau_{0}}\right)
$$

Lors d'une mesure CRDS, le temps de vie $\tau$ est mesuré en fonction de la pulsation de la radiation $\omega=2 \pi c / \lambda$, où $\lambda$ est la longueur d'onde. Ceci permet de tracer un spectre des pertes par passage $(d / c \tau)$ en fonction de $\omega$, dont la ligne de base correspond aux pertes liées aux coefficients de réflexion des miroirs (1 $R)$. En retranchant cette ligne de base, on obtient le spectre d'absorbance $k(\omega)$. Il est important de noter que la longueur d'absorbance $\ell$ n'est pas nécessairement égale à la distance entre les miroirs $d$. La méthode ne nécessite pas de connaître les coefficients de réflexion des miroirs. Lors des études en flamme ou dans les plasmas, le milieu réactif est placé au centre de la cavité optique [3,4], comme le montre la figure 2 .

\section{Sensibilité de la CRDS}

La puissance de la CRDS réside dans sa sensibilité. La technique permet d'une part de s'affranchir des fluctuations de l'intensité de la lumière incidente. D'autre part, le trajet optique dans la cavité augmente considérablement, et ceci d'autant plus que le traitement des miroirs est hautement réfléchissant. La limite de détection par absorption peut s'exprimer selon:

$$
\left[\frac{\alpha \ell}{d}\right]_{\min }=\frac{1-R}{d}\left(\frac{\Delta \tau}{\tau}\right)_{\min }
$$

Figure 2. Schéma de principe de la CRDS. R est le coefficient de réflexion des miroirs ; $d$, la distance entre les miroirs ; $\ell$, la longueur d'absorbance ; $c$, la célérité de la lumière ; $k$, l'absorbance.

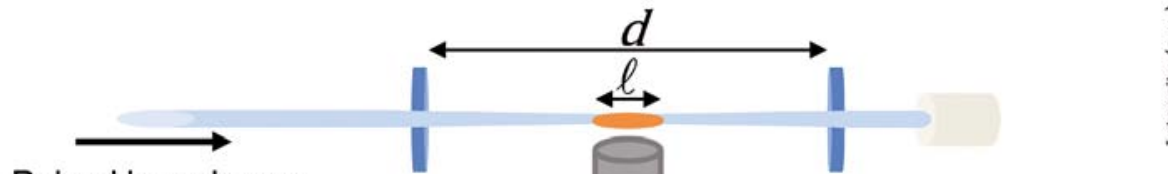

\section{Pulsed laser beam \\ flame \\ Photodetector}

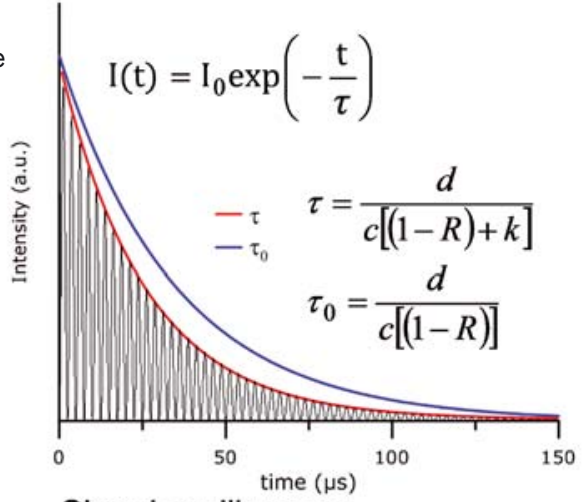

Signal oscilloscope 


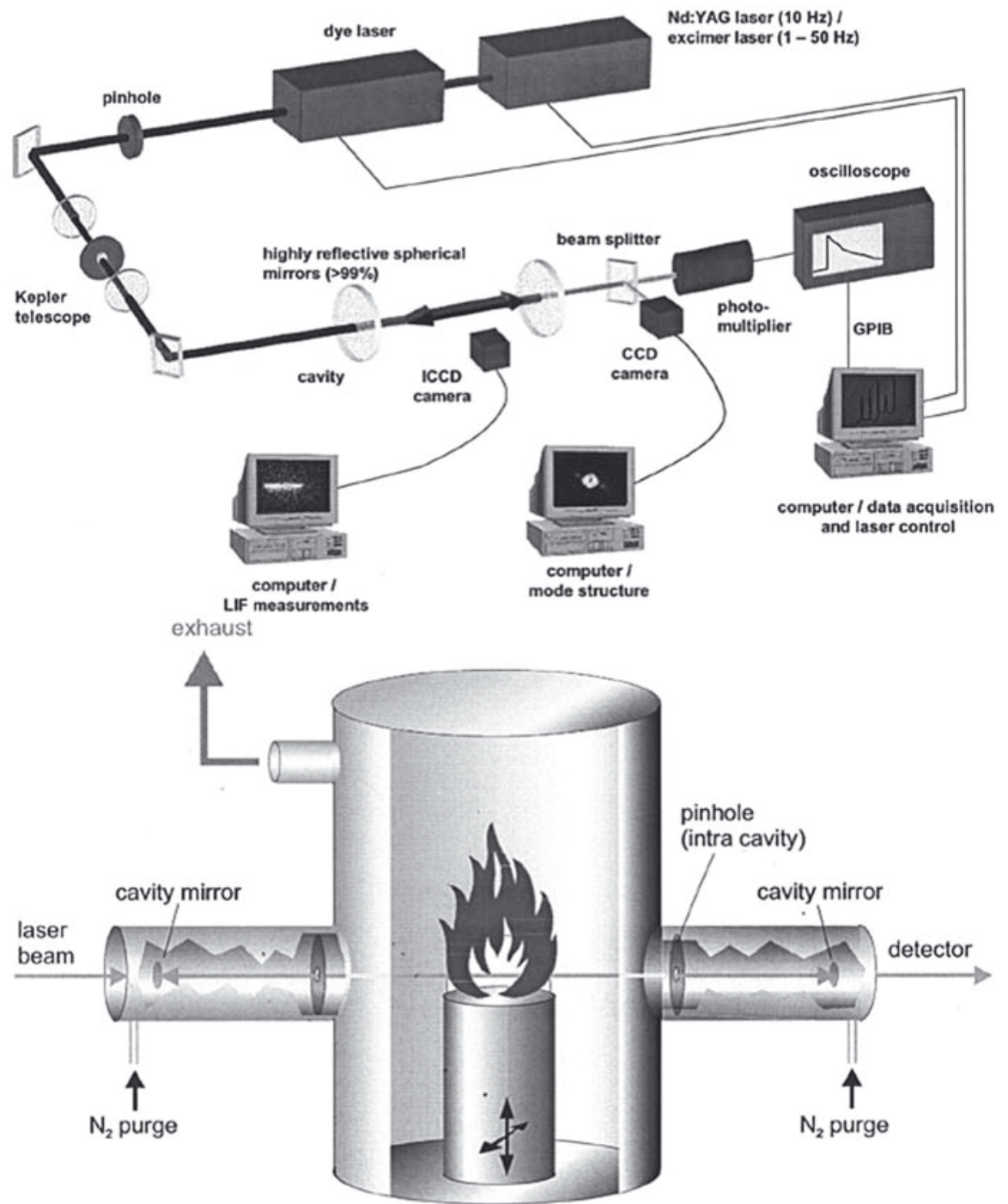

Figure 3. Dispositif expérimental de mesures CRDS dans une flamme, extrait de [3] avec la permission de The Optical Society.

Il est ainsi possible d'avoir une sensibilité de 3 à 4 ordres de grandeur supérieure à celle de l'absorption simple. Dans le domaine de l'infrarouge, la qualité de traitement des miroirs permet d'avoir des coefficients de réflexion supérieurs à 99,99\%. Cependant, dans le domaine du visible et de I'UV, les techniques de traitement ne permettent d'avoir des coefficients que de l'ordre de 99,5\% à $99,9 \%$. Cela se traduit par une sensibilité un peu moindre de la technique qui reste toutefois très élevée.

Prenons l'exempled'une cavité de $50 \mathrm{~cm}$, avec des miroirs de réflexion $R=99,8 \%$, la distance effective parcourue par le rayonnement est de $250 \mathrm{~m}$. Si la précision de mesure du temps de vie est de 0,5\%, le minimum détectable d'absorption est égal à $2 \cdot 10^{-5} \mathrm{~cm}^{-1}$. Si on prend des miroirs avec $R=99,99 \%$, le trajet effectif est égal à $5 \mathrm{~km}$ et le minimum détectable d'absorption est égal à $1.10^{-6} \mathrm{~cm}^{-1}$.

\section{Mise en œuvre de la CRDS pulsée en combustion}

Dans cet article, nous nous limiterons au cas de la CRDS pulsée. Cette technique est en effet mieux adaptée aux mesures réalisées dans des milieux à fort gradient de température (flammes, plasma, ...). Dans ce cas, comme l'illustre la figure 3, la flamme est située au centre de la cavité

\footnotetext{
Dans ce cas, si la distance d'absorption est égale à $5 \mathrm{~cm}$, et que l'espèce absorbante présente une section efficace d'absorption égale à $1.10^{-16} \mathrm{~cm}^{-1} /\left(\right.$ molécule $\mathrm{cm}^{-3}$ ) à $5 \mathrm{kPa}$ et $1500 \mathrm{~K}$, le minimum détectable en fraction molaire de l'espèce est égal à 0,4 ppm.
}

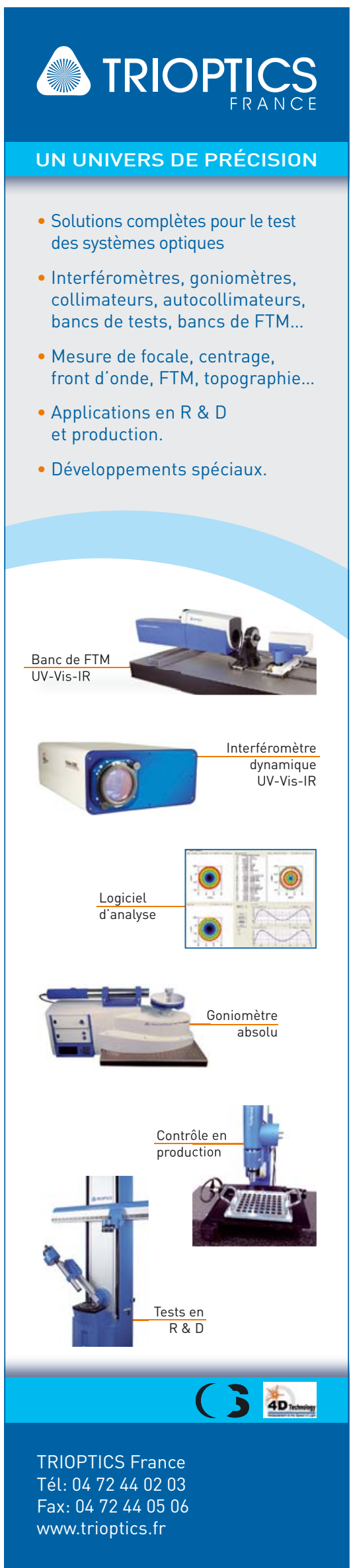

我 
optique. Le brûleur est mobile en translation (longitudinalement ou radialement). Comme toute mesure par absorption, la CRDS est une technique de mesure intégrée le long de l'axe laser. Dans ces applications, le mélange n'est pas homogène dans la cavité, mais on peut assimiler la longueur du volume de mesure associée au diamètre de la flamme.

\section{Les propriétés de la cavité optique}

La cavité optique doit présenter certaines spécificités qui sont bien décrites par Siegman [5]. Nous n'énonçons ici que les notions simplifiées.

(1) La cavité doit être optiquement stable. Le rayon de courbure des miroirs ( $r$ ) est choisi en fonction de la dimension de la cavité $(d)$ pour respecter $0<d<2 r$. En pratique, dans le cas de la CRDS, on dénombre 3 types de cavités.

- Les cavités confocales pour lesquelles la distance entre les miroirs est égale au rayon de courbure des miroirs $(d=r)$.

- Les cavités quasi-planes pour lesquelles le rayon de courbure des miroirs est infiniment grand devant la distance $d$ $(r>>d)$. Dans cette configuration, le diamètre du faisceau est quasi constant le long de l'axe longitudinal de la cavité. Cette configuration est souvent choisie pour les mesures réalisées dans une cellule d'absorption dans laquelle le mélange est homogène.

Les cavités quasi-concentriques pour lesquelles la distance entre les miroirs est voisine de $2 r$. Dans ce cas, le faisceau est très étroit au centre de la cavité. On préfèrera cette dernière configuration dans le cas où les mesures sont réalisées au centre de la cavité optique. Elle offre en effet la meilleure résolution spatiale.

(2) Elle doit être résonante de façon à transmettre l'intensité lumineuse selon la théorie de Fabry-Pérot. L'intervalle spectral libre (ISL ou FSR - free spectral range) défini par $c / 2 d$ correspond à l'intervalle en fréquence des modes de résonance de la cavité. Seules les fréquences du laser en résonance avec l'un des modes de la cavité sont transmises. Cette condition est vérifiée si la fréquence du laser ( $v$ ) est égale à un multiple entier de l'intervalle spectral libre.

\section{Injection de l'impulsion laser dans la cavité}

La structure de mode de la cavité peut poser différents problèmes. Lorsqu'on travaille avec une source laser pulsée, la largeur de raie laser est généralement très grande devant l'espacement des modes de la cavité. Dans ce cas, quelle que soit la fréquence d'excitation du laser, il y a toujours au moins un mode de transmission de la cavité qui est excité. Au contraire lorsqu'on travaille avec une source très fine spectralement (typiquement avec les sources continues), il faut adapter la dimension de la cavité à la fréquence d'excitation.

Il est par ailleurs préférable de s'assurer que seuls les modes transverses longitudinaux TEM ${ }_{00}^{2}$ de la cavité sont excités $[2,6]$. Pour cela, il convient de réaliser un montage optique généralement constitué de 2 lentilles et d'un pinhole pour injecter le laser selon les modes TEM ${ }_{00}$ de la cavité. Le dimensionnement du montage optique et de la cavité est régi par les lois de l'optique gaussienne. Cette mise en forme du faisceau laser permet par ailleurs d'améliorer la résolution spatiale du dispositif optique.

\section{Mesure de la décroissance temporelle}

Un détecteur photonique (photodiode ou photomultiplicateur) situé à la sortie de la cavité optique permet de mesurer l'intensité du signal transmis par celle-ci en temps réel. Le détecteur est associé à un oscilloscope ou une carte d'acquisition, en

${ }^{2} \mathrm{TEM}_{00}$ désigne le mode fondamental des modes électromagnétiques transverses (TEM) de la cavité laser. On parle également du mode gaussien. lien avec un ordinateur. Il est nécessaire de traiter en temps réel la détermination du temps de vie $\tau$ à partir de la mesure de la décroissance temporelle exponentielle du signal CRDS. Rappelons que la sensibilité de la technique est liée à la précision de la mesure de $\tau$. Il est, par exemple, important de ne pas intégrer dans le traitement numérique le bruit qui a un effet conséquent sur la détermination de $\tau$.

\section{Aspect quantitatif de la mesure par CRDS}

Lorsqu'on travaille en CRDS avec des sources laser accordables à colorant et pulsées (généralement présentant une largeur spectrale minimale de $0,05 \mathrm{~cm}^{-1}$ ), la largeur spectrale du laser est plus grande que l'intervalle spectral libre, assurant la transmission de l'intensité laser sur plusieurs modes comme le montre la figure 4.

En réalité, le signal lumineux est transmis par la cavité CRDS selon les modes longitudinaux $\omega_{\mathrm{m}}$. Chacun de ces modes subit une vitesse de perte propre, et le signal s'exprime par la somme des pertes individuelles sur les modes $\omega_{m}$ selon [7] :

$$
I(t, \omega)=\sum_{m} I_{0}(\omega) T\left(\omega_{m}\right) \exp \left[-t\left(1-R+k\left(\omega_{m}\right) l\right) \frac{c}{d}\right]
$$

Il faut prendre des précautions supplémentaires afin de s'assurer que le temps de déclin est représentatif $d$ 'une mesure quantitative. En effet, l'interprétation de la mesure repose sur une décroissance mono-exponentielle du temps de déclin.

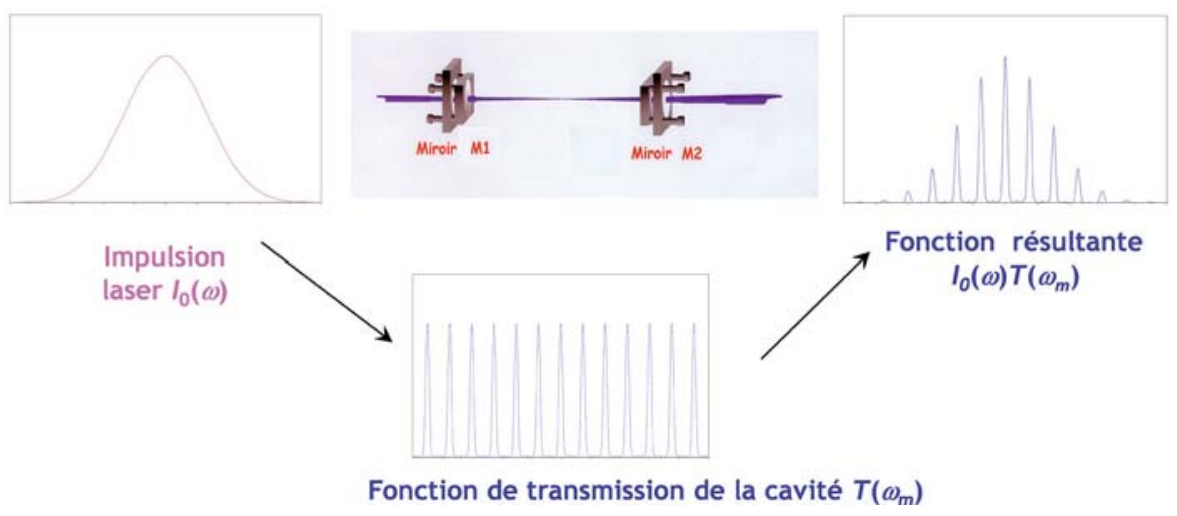

Figure 4. Modes de transmission d'une impulsion laser large spectralement (par exemple, un laser à colorant) dans une cavité optique CRDS. 

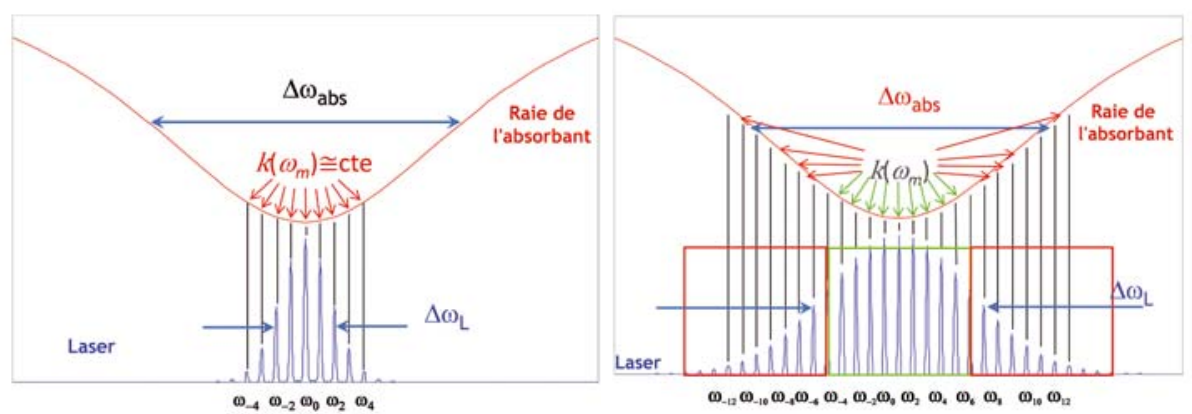

Figure 5. Modes de transmission de la cavité CRDS dans l'échelle des nombres d'onde dans un milieu absorbant centré sur $\omega_{0}$ lorsque $\Delta \omega_{\mathrm{L}} \ll \Delta \omega_{\text {abs }}$ (à droite) ou $\Delta \omega_{\mathrm{l}} \cong \Delta \omega_{\text {abs }}$ (à gauche).

Deux cas de figure peuvent se présenter comme le montre la figure 5.

- Si la raie laser $\left(\Delta \omega_{L}\right)$ est inférieure à la raie d'absorption de la molécule $\left(\Delta \omega_{\text {abs }}\right)$, on peut alors faire l'approximation que


ser sont affectés de la même vitesse de pertes dues à l'absorption de la molécule $\left(k\left(\omega_{\mathrm{m}}\right)\right.$ reste constante pour tous les $\omega_{\mathrm{m}}$ excités). La décroissance mono-exponentielle du temps de déclin de la cavité est alors vérifiée.

Si la raie laser se caractérise par une largeur similaire à celle de la raie d'absorption de la molécule, les modes de transmission centrés sur la raie d'absorption subissent une vitesse de perte plus importante que ceux situés en périphérie. Dans ce cas, le caractère mono exponentiel de la décroissance temporelle n'est plus garanti. Il est alors nécessaire de prendre en compte le caractère multi exponentiel de la décroissance temporelle du signal afin d'accéder à une mesure quantitative $[7,8]$.

\section{Application de la CRDS aux mesures en flammes}

De nombreux exemples d'applications de la CRDS pulsée pour des études en combustion sont répertoriés dans la littérature. Une synthèse des espèces détectées en flamme est reportée dans un ouvrage récent dédié à la technique CRDS [9].

En pratique, les pertes sont non seulement dues à la réflexion des miroirs et à l'absorption, mais également liées aux diffusions de Mie et Rayleigh dans la cavité. Ces pertes sont indépendantes de la longueur d'onde de la radiation laser et le principe de mesure du temps de vie hors absorption permet de déterminer l'ensemble des pertes non absorbantes.

\section{Obtention de spectres d'absorption}

La technique CRDS permet d'obtenir des spectres d'absorption. La mesure du temps de vie mesurée en fonction de la longueur d'onde (ou de la pulsation) est convertie en pertes par passage ou absorbance $(\alpha \ell)$ en assimilant la ligne de base à la mesure du temps de vie sans absorption. Une illustration d'un spectre du radical NCO mesuré à $2000 \mathrm{~K}$ est présentée à la figure 6. Ce spectre a été mesuré au sein d'une flamme avec une absorbance maximale $^{3}$ de 130 ppm, soit une atténuation de $2.10^{-5}$ par $\mathrm{cm}$. Dans ce travail, la flamme plate d'un diamètre de $6 \mathrm{~cm}$ était placée au centre d'une cavité optique de $42 \mathrm{~cm}$ de long, et la fraction molaire de NCO a été mesurée égale à 20 ppm.

\section{Analyse des mesures : détermination des concentrations d'espèces}

Si la section efficace d'absorption $\sigma(\omega)$ de l'espèce est connue, la mesure effectuée par CRDS comme toute mesure d'absorption permet également de déterminer la concentration absolue de l'espèce. On peut faire une mesure soit par absorptivité intégrée, soit au pic de la raie d'absorption. Dans le premier cas, lorsque la molécule présente un spectre de raies, on peut mesurer une des raies d'absorption de l'espèce en effectuant un balayage en longueur d'onde du laser autour de

${ }^{3}$ L'absorbance (ou pertes par passage) n'ayant pas d'unité, elle est souvent précisée en ppm.
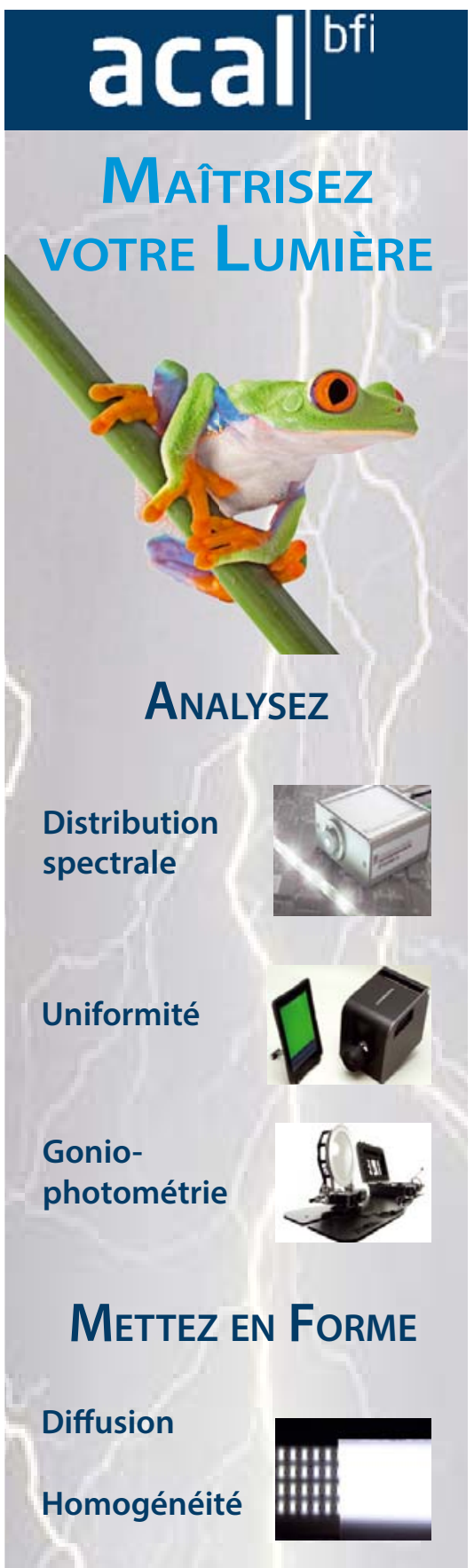

Piège

Absorption

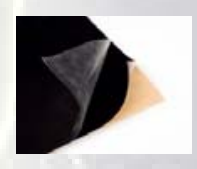

Focalisation

N'hésitez pas à nous solliciter $T+33$ (0)1 60795906 - photonique.fi@acalbfi.fr www.acalbfi.fr 


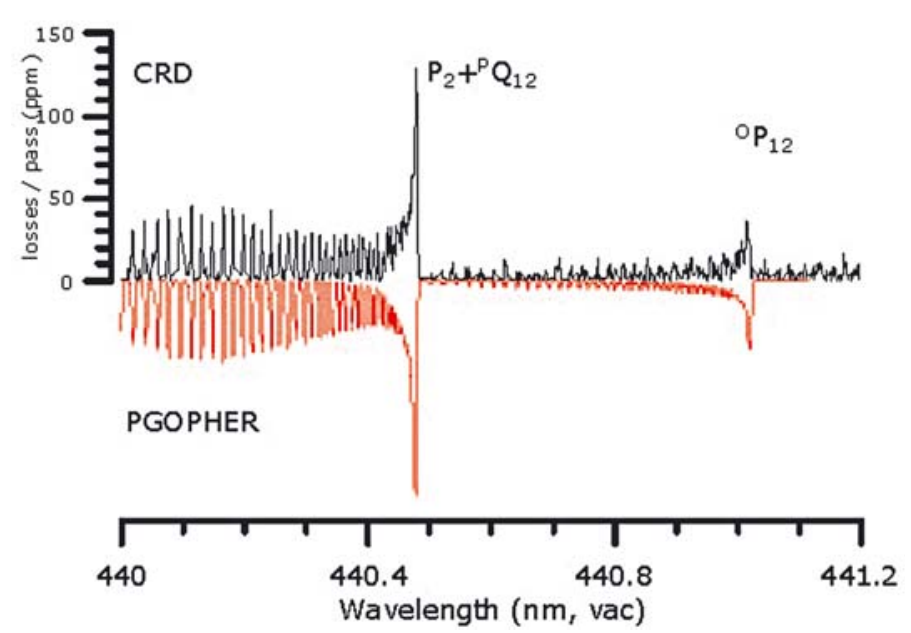

Figure 6. Comparaison entre un spectre simulé et un spectre expérimental de NCO mesuré dans une flamme à une température de $2000 \mathrm{~K}$ [10].

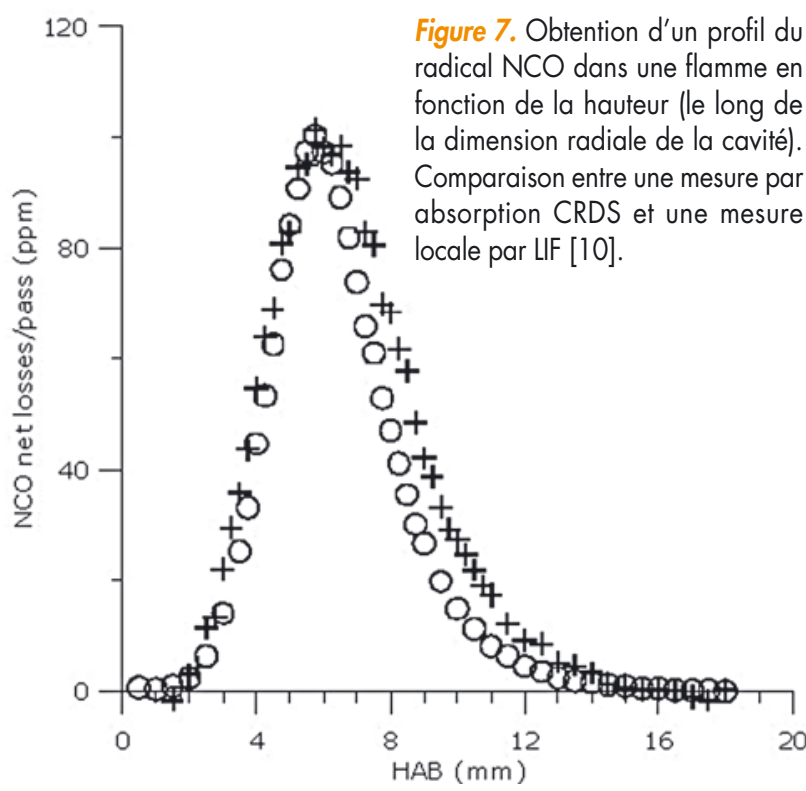

celle-ci. La détermination de l'absorptivité intégrée permet de déterminer la concentration de l'espèce à partir de la force de raie $I\left(\omega_{0}\right)$ et ne requiert pas de connaître la largeur spectrale du laser, selon :

$A(\omega)=\int \sigma(\omega) N \ell d \omega=I\left(\omega_{0}\right) N \ell=f_{B}\left(T, \omega_{0}\right) h b \omega_{0} N \ell$

$f_{B}\left(T, \omega_{0}\right)$ est le facteur de Boltzmann à la température $T, h$ est la constante de Planck, $b$ est le coefficient d'Einstein pour l'absorption à la transition $\omega_{0}$ (normalisé par $c)$.

Dans le deuxième cas, on fait successivement la mesure du temps de vie au pic de la raie d'absorption et à une longueur d'onde hors absorption pour déterminer les pertes par absorption $k\left(\omega_{0}\right)$ :

$$
k\left(\omega_{0}\right)=\sigma\left(\omega_{0}\right) N \ell=I\left(\omega_{0}\right) g\left(\omega_{0}\right) N \ell
$$

Il faut déterminer la fonction d'élargissement laser $g(\omega)$ (avec $\int g(\omega) d \omega=1$ ).

On fait l'hypothèse que le mélange est homogène le long du diamètre de la flamme, $\ell$. Celle-ci peut être vérifiée par comparaison des mesures relatives réalisées localement par fluorescence induite par laser (LIF, laserinduced fluorescence) et des mesures par absorption CRDS comme le montre la figure 7. Notons que si dans le volume de mesure, le mélange n'est pas homogène mais présente au moins un axe de symétrie, on peut traiter la mesure de l'absorption par une inversion d'Abel.

\section{Performances et variantes de la CRDS}

La performance de la CRDS pulsée a été démontrée, et permet de mesurer des espèces traces avec une sensibilité de l'ordre de $10^{-6} \mathrm{~cm}^{-1}$. Cette technique, relativement simple à mettre en œuvre, est applicable aux mesures dans des milieux complexes tels que ceux rencontrés dans les études en combustion ou plasma, avec la présence d'un fort gradient thermique. Cependant, avec les sources pulsées, plusieurs modes longitudinaux de la cavité sont excités et cela entraîne des limitations de la sensibilité. L'une des premières variantes de la CRDS est la cw-CRDS. Dans ce cas, les sources continues qui offrent généralement une largeur spectrale fine $\left(10^{-2} \mathrm{~cm}^{-1}\right)$ permettent de n'exciter qu'un seul mode de la cavité. La sensibilité de la cw-CRDS est de l'ordre de $10^{-9} \mathrm{~cm}^{-1}$. Cependant, la mise en œuvre de cette technique est plus délicate (optimisation de la longueur de la cavité avec la fréquence de la source) et n'est applicable que dans des cellules homogènes.

Pour en savoir plus sur la technique CRDS et ses variantes, nous recommandons l'ouvrage édité par Berden et Engeln [9].

L'auteur souhaite exprimer ses remerciements à ses collègues Pascale Desgroux et Xavier Mercier.

\section{Références}

[1] S. Kassi, D. Romanini, Spectromètre laser à amplification résonante d'absorption. Applications à la mesure de trace de gaz, Tech. Ing. RE-100 (2008)

[2] A. O'Keefe, D.A.G Deacon, Cavity RingDown spectrometer for absorption measurements using pulsed laser sources, Rev. Sci. Instr. 59(12) (1988) 2544-2551

[3] A. Schocker, K. Kohse-Hoïnghaus, A. Brockhinke, Quantitative determination of combustion intermediates with cavity ring-down spectroscopy: systematic study in propene flames near the soot - formation limit, Appl. Opt. 44(31) (2005) 6660-6672

[4] G.D. Stancu, F. Kaddouri, D.A. Lacoste, C.O. Laux, Atmospheric pressure plasma diagnostics by OES, CRDS and TALIF, J. Phys. D: Appl. Phys. 43(12) (2010) 124002

[5] A. Siegman, Lasers, University Siences Books, Mill Valley, CA (1986)

[6] D. Romanini, A.A. Kachanov, F. Stoeckel, Cavity ringdown spectroscopy: broad band absolute absorption measurements, Chem. Phys. Lett. 270(5-6) (1997) 546-550

[7] P. Zalicki, R.N. Zare, Ring-Down Spectroscopy for absorption measurements, J. Chem. Phys. 102(7) (1995) 2708-2717

[8] X. Mercier, E. Therssen, J.F. Pauwels, P. Desgroux, Comb. Flame 124(4) (2001) 656-667

[9] G. Berden, R. Engeln, Cavity Ring-Down Spectroscopy: Techniques and Applications, John Wiley and Sons, Inc. 